\title{
Measurement and Analysis of Dielectric Properties of Agricultural By-product Powders in Microwave Frequency Range
}

\author{
Runeng Zhong ${ }^{1}$, Tai Xiang ${ }^{1}$, Qinhong Zheng ${ }^{1,2^{*}}$, Bo Xu ${ }^{1}$ \\ ${ }^{1}$ School of energy and environmental science, Yunnan Normal University, Kunming 650500, PR China \\ ${ }^{2}$ Key Laboratory of Photoelectric Information Technology of Yunnan Province, Yunnan Normal University, Kunming 650500, China
}

\begin{abstract}
Dielectric properties play an important role in the microwave-assisted application of nonmagnetic agriculture-related media. In this paper, the practical requirements for the lack of quantitative data on the dielectric properties of agricultural media in microwave-assisted applications are obtained. The dielectric properties data of four kinds of agricultural by-products powder (Potato powder, Pseudo -ginseng Powder, Gastrodia elata powder, Dendrobium chrysotoxum Lindl powder) under microwave frequency were measured, and related factors were analyzed. The results show that the uncalibrated coaxial transmission reflection method can be used to measure and analyse the dielectric properties of high frequency bands of powder materials. Dielectric constant $\left(\varepsilon^{\prime}\right)$ and Dielectric loss factor $\left(\varepsilon^{\prime \prime}\right)$ of agricultural by-products at room temperature have a relationship between microwave frequency and moisture content. It is a dependent and the mathematical relation is satisfied. The influence of microwave frequency and moisture content on the dielectric properties is as follows: $\varepsilon^{\prime}>\varepsilon^{\prime \prime}>\tan \delta$. Under the conditions of civil microwave frequency $(2.45 \mathrm{GHz})$ and moisture content $(2 \% \sim 25 \%)$, the dielectric constant and the dielectric loss factor of different kinds of agricultural by-products powder are in the range of $3.5 \sim 9$ and $0.1 \sim 3.2$ respectively. The results provide data basis for microwave processing and quality monitoring of agricultural by-products powder. The technical method is suitable for the measurement and analysis of the dielectric properties of powder materials.
\end{abstract}

\section{INTRODUCTION}

Dielectric properties are the basis of microwave-assisted application research of non-magnetic agriculture-related media. In the past few decades, many scholars have focused on the requirements of thermal processing, storage, preservation, sterilization, grading and nondestructive testing of agricultural media. Different technical methods were applied to study the dielectric properties and influencing factors of agricultural media ${ }^{1-}$ ${ }^{4}$. The research results strongly promoted the microwave thermal processing application and product development of agricultural media. Among the various types of agricultural-related media, powdered agricultural byproducts after primary processing have been widely welcomed by people because of their convenience electric properties of agricultural media. The measurement targets are mainly concentrated on fresh vegetables, fruits, starch water systems with high dielectric constant and a small number of main food crops (wheat, rice, etc.) ${ }^{5-9}$. Some scholars carried out experimental measurements of dielectric properties of Red pepper powder ${ }^{4}$, Aspen powder ${ }^{10}$, Lactose ${ }^{11}$, Pseudo -ginseng Powder ${ }^{12}$. There are very few types and quantities. The technical method adopted is mainly based on the probe method, the cavity method and the lumped parameter method for measuring high dielectric constant, so it is mainly focused on the measurement and analysis of the dielectric properties of the low frequency band of the medium ${ }^{13}$. In general, there is a relative lack of quantitative data on the dielectric properties of different types of agricultural by-products. In particular, the measurement of the dielectric properties of powders of Chinese drug and food homology is basically in the blank, and the marketization of microwave-assisted applications of agricultural by-products. Scale is still far from being scaled to suit the needs. Therefore, this paper uses a non-calibrated coaxial transmission reflection method with a wide frequency measurement range to experimentally measure the dielectric properties of agricultural by-products (potato powder, Pseudo ginseng Powder, Gastrodia elata powder, Dendrobium chrysotoxum powder) under microwave frequency range. The interdependence of dielectric constant and dielectric loss factor of different kinds of agricultural by-products on microwave frequency and water content provides a technical method for the measurement of powder dielectric properties of different agricultural by-products, and provides a basis for microwave assisted applications.

\footnotetext{
* Corresponding author: Qinhong Zheng, 76519159@qq.com.
} 


\section{MATERIALS AND METHOD}

\subsection{Material}

The four types of agricultural (secondary) products were purchased from stores, and the raw materials were produced in Yunnan province of China. They were potato powder, dendrobium chrysotoxum powder, gastrodia elata powder and pseudo -ginseng powder. The moisture content under normal temperature was $10.2 \%$, $11 \%, 10.2 \%$ and $9 \%$ respectively. Experimental paraffin powder (density $0.87 \mathrm{~g} / \mathrm{cm} 3$ ) and deionized water were provided by Yunnan bioengineering research centre.

\subsection{Instrument and Equipment}

ZNB20 vector network analyze (R\&S, Munich, Germany); 85051B 7mm / APC-7 coaxial air line (Agilent Technology, Penang, Malaysia); moisture meter (Changzhou Hengdian Co., Ltd., weighing accuracy $0.005 \mathrm{~g})$.

\subsection{Experimental Method}

\subsubsection{Preparation of Sample}

At room temperature $(24 \pm 1){ }^{\circ} \mathrm{C}$, the paraffin wax is heated to 70 degrees, which was melted and mixed with agricultural by-products powder, deionized water. It was fully and uniformly stirred and mixed using an automatic stirrer. When it returned to room temperature and then placed into the sample molding device, it was pressed to obtain a mixture of ring columnar sample. It uses vernier caliper to obtain sample length value and record, weigh, calculate volume fraction. It has the record, vacuum packaging, number stored in a $9{ }^{\circ} \mathrm{C}$ environment for testing. We had the preparation of powder/paraffin mixture samples of agricultural by-products with different water contents.

\subsubsection{Determination of moisture content}

The moisture content was determined according to the drying method principle, and three parallel experiments were performed on each group of samples by using a moisture analyzer to ensure that the mass difference was less than $5 \mathrm{mg}$. The arithmetic mean value was taken as the measured value.

\subsubsection{Measurement System}

We use the APC-7 3mm adapter and coaxial cable to connect the 85051B coaxial transmission line fixture to the two ports of the ZNB20 vector network analyzer, start preheating the ZNB20 network analyzer, and start the network analyzer S parameter measurement system after $1 \mathrm{~h}$. calibration). When measuring, we set the measurement frequency to $2-5 \mathrm{GHz}$ according to the operation specification, select 150 frequency points at equal intervals, set 20 repeated scans for each measurement, and take the arithmetic mean as the measured value.

\subsubsection{Measurement of scattering parameters}

The sample to be tested is placed at room temperature $(24 \pm 1){ }^{\circ} \mathrm{C}$ for about $5 \mathrm{~h}$, and the sample temperature is returned to room temperature. The sample of the mixture to be tested is placed in the coaxial transmission line. After stabilization, the scattering parameters are measured and saved, the sample is taken out for weighing, recording, and the measuring instrument is cleaned. The measurement of the same sample was repeated 3 times and its arithmetic mean was taken.

\subsection{Calculation of Dielectric Properties}

\subsubsection{Calculation of Dielectric Properties of Mixture Samples}

Based on the measurement principle of uncalibrated coaxial transmission line method ${ }^{14}$, the mathematical equation of the measured value of $\mathrm{S}$ parameter and the dielectric characteristics of the mixture to be tested was deduced from the electromagnetic field theory and $\mathrm{T}$ matrix transformation theory. The equation was solved by using Newton iterative numerical calculation method. Matlab software was used to compile relevant programs for calculation, and the complex relative permittivity values describing the dielectric characteristics of the mixture under test were obtained.

\subsubsection{Calculation of Volume Fraction}

The volume fraction of powder material is equal to the ratio of the volume of powder in the sample to the total volume of the mixture sample ${ }^{15}$. $f_{v}=1-\left(m_{s} \times\left(m_{1} / m_{2}\right)\right) /(\rho \times \mathrm{V})$. Where $V=\pi\left(b^{2}-a^{2}\right) l / 4$, $f_{v}$ is the volume fraction, $m_{s}$ is the mass of paraffin in sample preparation and $m_{1} 、 V 、 l$ are respectively the total mass, volume and length of the mixture sample, $m_{2}$ is the mass of the agricultural product powder in sample preparation. $\rho\left(0.87 \mathrm{~g} / \mathrm{cm}^{3}\right)$ is the density of paraffin, and $a(=3.04 \mathrm{~mm}), b(=7 \mathrm{~mm})$ is the inner diameter and outer diameter of the ring columnar sample respectively.

\subsubsection{Calculation of powder dielectric properties to be measured}

According to the principle of power exponent method, the dielectric properties of powder materials are calculated. $\varepsilon_{\text {eff }}^{\beta}=f \varepsilon_{i}^{\beta}+(1-f) \varepsilon_{e}^{\beta}$.in which, $\varepsilon_{\text {eff }}$ is complex dielectric property of mixtures. $\varepsilon_{i}$ and $\varepsilon_{e}$ are dielectric property of agricultural products powder and paraffin powder, $f$ is the volume fraction of the powder for agricultural by-products. $\beta$ is a dimensionless parameter. When $\beta=1 / 2$, there is Brichak formula ${ }^{16}$. In this paper, Brichak had its formula calculation using matlab 
software programming for solving. The calculation index is dielectric constant $\varepsilon_{i}^{\prime}$ and dielectric loss factor $\varepsilon_{i}^{\prime \prime}$.

\subsection{Data Processing and Analysis}

Using the data processing software Matlab R2012a(Math Works,Massachusetts State), Origin 8.5(Atos Origin, Amsterdam, Netherlands), we had all excel conduct data processing and linear regression analysis.

\section{EXPERIMENTAL RESULTS AND ANALYSIS}

\subsection{Verification of Measurement Scheme}

In order to verify the correctness of the measurement scheme, the experiment measured the circle columnar paraffin sample (length of $38 \mathrm{~mm}$ ), the scattering parameters of average after repeated measurement is three times. The program calculation is according to section Brichak formula for measuring dielectric properties of paraffin wax powder, paraffin dielectric constant is $\varepsilon^{\prime}=2.238 \pm 0.02$, and dielectric loss tangent value is 0.0031 . The calculation coincides with the experimental value ${ }^{15}$. To test and verify the rationality and accuracy of Brichak formula, selection of dry snow air mixture $\left(\varepsilon_{i}=3.15-j 0.001, \varepsilon_{e}=1\right)$ is as the object. The numerical results of different theoretical formula to calculate the comparison with measured values ${ }^{17}$ literature. Graphic shows that adopting Brichak formula to get the numerical results with the literature the highest measured values coincide and Bruggeman lichtenecker formula. Accordingly, we select Brichak formula as agricultural by-products formula powder material dielectric properties.

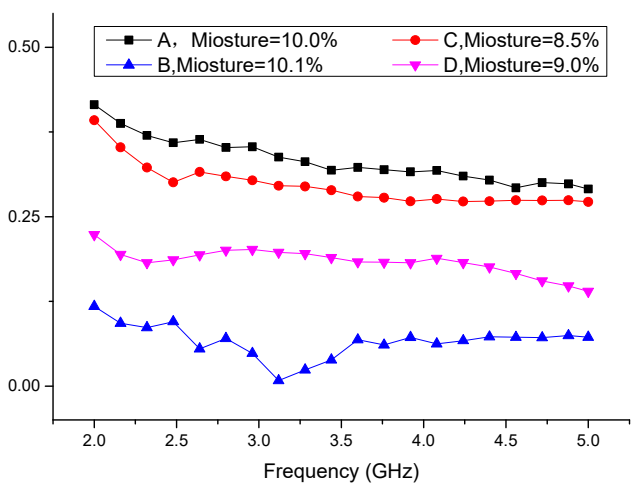

(b)

Fig1. Changes of dielectric property of 4 agricultural by-products with microwave frequency, (a) dielectric constant (b) dielectric loss factor

\subsection{Relationship between dielectric characteristics and microwave frequency}

When room temperature is able to make potato powder/paraffin wax powder mixture samples, dendrobium chrysotoxum powder/paraffin wax powder mixture samples, gastrodia elata powder/paraffin wax powder mixture samples and pseudo-ginseng Powder/paraffin wax powder mixture samples, in turn, marked as A, B, C, D, and moisture content of $10.2 \%$, $11 \%, 10.2 \%$ and $9 \%$ respectively. According to section 1.4.2, calculated volume fractions of powder mixture in the corresponding were $0.518,0.410,0.577,0.431$, the selection of microwave frequency range as the $2 \sim 5 \mathrm{GHz}$ and measured. According to the theory in section 1.4.1. Matlab program was prepared to realize the conversion calculation of the mixture's electromagnetic scattering parameters and dielectric property, and the program was compiled according to section 1.4.3 Brichak formula to calculate the dielectric property of the powder to be tested. Figure 1 shows the four kinds of agricultural byproducts of the dielectric constant and loss factor with the change of microwave frequency, graphic show that: (1) with the increase of frequency, dielectric constant of the same moisture content of powder material is monotone decreasing trend. The dielectric loss factor is rising and falling, the change trend and cavity method, which is used to measure the dielectric parameters of winter wheat consistent trends ${ }^{13}$. (2)The dielectric properties of different powder substances are different. As can be seen from figure 1 (a), $\varepsilon_{A_{-} 10 \%}>\varepsilon_{C_{-} 8.5 \%}>\varepsilon_{D_{-} 9 \%}>\varepsilon_{B_{-} 10.1 \%}$. The moisture content of the dendrobium chrysotoxum powder (B) is similar to that of potato powder (A), the dielectric properties of the two substances are different, which may be related to the microwave penetration ability and the shape and size of the powder substance in the sample.

\subsection{Relationship between dielectric Properties and moisture content}

The dielectric properties of four kinds of substances under different moisture content were measured by using the civil microwave frequency $(2.45 \mathrm{GHz})$ as the 
observation point. Figure.3, figure 4 shows the dielectric constant $\left(\varepsilon^{\prime}\right)$ and loss factor $\left(\varepsilon^{\prime \prime}\right)$ of different moisture content of agricultural by-products powder at $2.45 \mathrm{GHz}$ respectively. The diagram shows that:

(1) The dielectric properties of the same powdered substances increased with the water content, but the range of the dielectric properties of different kinds of powder substances varied with the moisture content. On the whole, in the range of common moisture content of dry matter of agricultural products $M \in(2 \%, 25 \%)$, the dielectric constant $\left(\varepsilon^{\prime}\right)$ of agricultural by-products powder is in the range of $3.5 \sim 9$, and the loss factor $\left(\varepsilon^{\prime \prime}\right)$ is between $0.1 \sim 3.5$. This macroscopic phenomenon can be used as a reference for the design of microwave processing devices for agricultural by-products powder.

(2) It is difficult to use a fixed calibration equation to predict the exact relationship between the dielectric properties and moisture content of all powder materials. In order to predict the dielectric properties of powder materials from macroscopic perspective, the average dielectric properties of four kinds of powder substances under the same water content $(\mathrm{M})$ were fitted linearly, and the dielectric properties of powder substances characterized by $2.45 \mathrm{GHz}$ were obtained. The normal equations of the relationship between dielectric constant, loss factor and moisture content are as follows: $\varepsilon^{\prime}=4.5388-0.1367 M+0.0106 M^{2} \quad\left(\quad \mathrm{R}^{2}=0.956\right)$, $\varepsilon^{\prime \prime}=0.0637-0.1250 M+0.0082 M^{2} \quad\left(\mathrm{R}^{2}=0.986\right)$. The above equations can be used to predict the numerical range of dielectric properties of powder materials.

\subsection{Relationship between dielectric Properties and Microwave Frequency and moisture content}

In order to study the relationship between the dielectric properties of different kinds of agricultural by-products powder and microwave frequency, moisture content at room temperature, we studied samples with different moisture content, which were prepared by 2.3.1 section method. The basic information of the samples is shown in Table 1.

The scattering parameters of the above samples were measured and calculated in turn. The dielectric properties and moisture content of the four kinds of powder substances to be measured. The diagram shows:

(1) Under the conditions of microwave frequency $(2-5 \mathrm{GHz})$ and water content $\mathrm{M}(5-25 \%)$, the dielectric constant $\left(\varepsilon^{\prime}\right)$, dielectric loss factor $\left(\varepsilon^{\prime \prime}\right)$ and loss angle tangent $(\tan \delta)$ of different kinds of agricultural by-products powder are dependent on frequency $(F)$ and water content(M).

Table 1 Basic information of four kinds of powder samples under different moisture content (M\%)

\begin{tabular}{cccccccc}
\hline $\begin{array}{c}\text { Potato } \\
\text { (A) }\end{array}$ & $\begin{array}{c}\text { Dendrobium } \\
\text { chrysotoxum } \\
\text { (B) }\end{array}$ & \multicolumn{2}{c}{$\begin{array}{c}\text { Gastrodia } \\
\text { elata }\end{array}$} & \multicolumn{2}{c}{$\begin{array}{c}\text { Pseudo- } \\
\text { ginseng } \\
\text { (D) }\end{array}$} \\
\hline $\mathrm{M}$ & $f_{v}$ & $\mathrm{M}$ & $f_{v}$ & $\mathrm{M}$ & $f_{v}$ & $\mathrm{M}$ & $f_{v}$ \\
\hline 5 & 0.500 & 10 & 0.311 & 8.5 & 0.560 & 9 & 0.427 \\
10 & 0.518 & 15 & 0.334 & 10.2 & 0.577 & 13 & 0.431 \\
14.7 & 0.528 & 21.5 & 0.368 & 17 & 0.582 & 19 & 0.469 \\
21.3 & 0.541 & 24.6 & 0.383 & 23.6 & 0.604 & 26 & 0.470 \\
\hline
\end{tabular}

(2) The relationship between dielectric constant $\left(\varepsilon^{\prime}\right)$, loss factor $\left(\varepsilon^{\prime \prime}\right)$, tangent of loss angle $(\tan \delta)$ and microwave frequency $(F)$, water content $(\mathrm{M})$ of four kinds of powdered substances $(A, B 、 C 、 D)$ in fig. 5 are fitted by polynomial, and the calculation equations of dielectric properties of different materials under $F$ (2-5GHz) and M (5-25\%) are obtained. The corresponding mathematical equations are shown in Table 2.

Order to analyse the relationship between dielectric constant $\left(\varepsilon^{\prime}\right)$, loss factor $\left(\varepsilon^{\prime \prime}\right)$, tangent of loss angle $(\tan \delta)$ and microwave frequency $(F)$, water content

(M) of four kinds of powdered substances (A、B、C、 D) in fig. 5 are fitted in a straight line. The slope of the fitting line is used to characterize the amplitude of the parameters varying with the frequency. The results show that in the range of water content $\mathrm{M}(5-25 \%)$, the range of the measured value of powder material and the slope value of the frequency fitting line $\varepsilon^{\prime}, \varepsilon^{\prime \prime}$ and $\tan \delta$ are respectively, $\quad k_{\varepsilon^{\prime}} \in(-0.42,-0.01)$ $k_{\tan \delta} \in(-0.004,-0.001)$.The results show that the influence of frequency and moisture content on the dielectric properties of powder is as follows: $\varepsilon^{\prime}>\varepsilon^{\prime \prime}>\tan \delta$. The change of water content and ratio of water had the greatest influence on the dielectric constant $\left(\varepsilon^{\prime}\right)$, followed by the loss factor $\left(\varepsilon^{\prime \prime}\right)$. However, $k_{\tan \delta}$ the slope of the fitted straight line $\tan \delta$ and $F$ is basically unchanged, indicating that there is little difference in the coupling ability between different powder materials and microwave field.

\section{CONCLUSIONS}

The dielectric characteristic data of four kinds of agricultural by-products powder at microwave frequency (room temperature) were measured by uncalibrated coaxial transmission reflection method. The results are as follows:

Table 2. Mathematical relationship between dielectric properties and frequency and moisture content of four kinds of powdered substances are studied.

\begin{tabular}{cccc}
\hline $\begin{array}{c}\text { mater } \\
\text { ial }\end{array}$ & $\begin{array}{c}\text { dielectric } \\
\text { properties }\end{array}$ & Mathematical equation & $R^{2}$ \\
\hline & $\varepsilon^{\prime}$ & $\varepsilon_{A}^{\prime}=4.588-0.062 F+0.013 M-0.021 F M+0.019 F^{2}+0.009 M^{2}$ & 0.976 \\
A & $\varepsilon^{\prime \prime}$ & $\varepsilon_{A}^{\prime \prime}=0.356-0.017 F-0.055 M-0.009 F M+0.013 F+0.008 M^{2}$ & 0.997 \\
& $\tan \delta$ & $\tan \delta_{A}=0.057-7.317 e^{-3} F-3.354 e^{-3} M-3.781 e^{-4} F M+1.725 e^{-3} F^{2}+7.538 e^{-4} M^{2}$ & 0.988 \\
B & $\varepsilon^{\prime}$ & $\varepsilon_{B}^{\prime}=3.517-0.099 F-0.016 M-0.016 F M+0.029 F^{2}+0.006 M^{2}$ & 0.991
\end{tabular}




$$
\begin{array}{cccc} 
& \varepsilon^{\prime \prime} & \varepsilon_{B}^{\prime \prime}=0.327+0.064 F-0.081 M-0.006 F M+1.608 e^{-2} F^{2}+5.237 e^{-3} M^{2} & 0.997 \\
& \tan \delta & \tan \delta_{B}=0.011+6.501 e^{-3} F-6.172 e^{-3} M-3.65 e^{-4} F M-2.99 e^{-4} F^{2}-6.30 e^{-4} M^{2} & 0.994 \\
\varepsilon^{\prime} & \varepsilon_{C}^{\prime}=3.791-0.3191 F+0.0609 M-0.0553 F M+0.1223 F^{2}+0.0107 M^{2} & 0.980 \\
\mathrm{C} \quad \varepsilon_{C}^{\prime \prime}=0.481+-0.065 F-0.043 M-0.025 F M+0.045 F^{2}+7.992 e^{-3} M^{2} & 0.994 \\
& \varepsilon^{\prime \prime} & \tan \delta_{C}=0.023-0.017 F+0.013 M+3.473 e^{-4} F M+1.256 e^{-3} F^{2}+4.367 e^{-6} M^{2} & 0.990 \\
\tan \delta & \varepsilon_{D}^{\prime}=4.418-0.214 F-0.079 M-1.247 e^{-2} F M+0.041 F^{2}+7.462 e^{-3} M^{2} & 0.967 \\
\varepsilon^{\prime} & \varepsilon_{D}^{\prime \prime}=1.109-8.459 e^{-2} M-0.130 F-2.563 e^{-4} F M+1.014 e^{-2} F^{2}+5.782 e^{-3} M^{2} & 0.967 \\
\varepsilon^{\prime \prime} & \tan \delta_{D}=0.163-0.015 M-0.015 F+6.323 e^{-4} F M+6.704 e^{-4} F^{2}+6.602 e^{-4} M^{2} & 0.954
\end{array}
$$

( 1 ) The uncalibrated coaxial transmission reflection method based on $\mathrm{T}$ matrix can be used to measure and analyze the dielectric properties of powder materials in high frequency band, which can effectively avoid the fixture calibration in the traditional transmission reflection method. It is difficult to measure and determine the location of multi-sample.

(2) The dielectric properties of agricultural product powder and microwave frequency and water content are interdependent to some extent. With the increase of frequency, the relative dielectric constant $\left(\varepsilon^{\prime}\right)$ of the same moisture content agricultural by-product powder shows monotonically decreasing trend. Loss factors $\left(\varepsilon^{\prime \prime}\right)$ may increase or decrease. With the increase of water content, the dielectric constant $\left(\varepsilon^{\prime}\right)$ and loss factor $\left(\varepsilon^{\prime \prime}\right)$ of agricultural by-product powder at the same frequency increased monotonically. The influence of microwave frequency and water content on the dielectric characteristics of agricultural products is as follows: $\varepsilon^{\prime}>\varepsilon^{\prime \prime}>\tan \delta$ 。

( 3 ) At the civil microwave frequency point $(2.45 \mathrm{GHz})$, the dielectric properties of different agricultural by-products powder vary with moisture content, so it is difficult to use a fixed calibration equation to characterize the exact relationship between the dielectric properties and moisture content of all powder materials. In the range of common moisture content of dry matter of agricultural products (5-25\%), the dielectric constant $\left(\varepsilon^{\prime}\right)$ of powdered agricultural byproducts are between $3.5 \sim 9$. The loss factor $\left(\varepsilon^{\prime \prime}\right)$ is between $0.1 \sim 3.5$. This macroscopic phenomenon can be used as a reference for the design of microwave hot processing device for powdered agricultural products.

( 4 ) On the basis of the measured data, the mathematical expressions of dielectric properties, moisture content and microwave frequency of different kinds of powdered agricultural (or by-products) were obtained by fitting, which provided the basis for the microwave identification, microwave sterilization, microwave drying and other microwave-assisted applications and device design of different powdered agricultural (or by-products).

\section{Acknowledgment}

Fund project: This research project was financially supported by the National Natural Science Foundation of China (Nos.31560305) and Science and Technology
Plan Project in Yunnan Province, Fund Number: 2016 FB141.

\section{References:}

1. Nelson, S. O.; Trabelsi, S. Dielectric Properties of Agricultural Products; Springer Netherlands, 2011.

2. Chee, G.; Rungraeng, N.; Han, J. H.; Jun, S. Journal of Food Science 2014, 79, E195-E201.

3. Shrestha B, B. O. D. Biosystems Engineering 2013, 114, 195-204.

4. GUO, W.; ZHU, X. Food \& Bioprocess Technology 2014, 7, 3591-3601.

5. Nelson, S. Dielectric Properties of Agricultural Materials and their Applications, 2015.

6. Sharma G P, P. S. Journal of Food Engineering, 2002, 52, 343-348.

7. Pace, W. E.; Westphal, W. B.; Goldblith, S. A.; Dyke, D. Journal of Food Science 2010, 33, 37-42.

8. FAN G, W. L. Food Science 2017, 38, 80-85. (in Chinese with English abstract).

9. S, H.; D, Z.; JS, C. food \& machinery 2011, 27, 165-167.

10. McMinn, W. A. M.; McLoughlin, C. M.; Magee, T. R. A. Drying Technology 2005, 23, 513 - 532.

11. Wam, M. M. Journal of Food Engineering 2006, 72, 113123.

12. Y, Q.; W, Y. Journal of Kunming University of Science and Technology 2016, 10, 81-87.

13. Nelson, S. O.; Trabelsi, S. Journal of Microwave Power and Electromagnetic Energy 2016, 50, 237 - 268.

14. Zhao C, J. Q. EEE Transactions on Electromagnetic ompatibility, 2011, 53(3):684-689.

15. L, H.; E, L.; G, G. Progress in Electromagnetics Research Letters 2013, 37, 177-187.

16. Elena Ciomaga, C.; Stefania Olariu, C.; etal. Journal of Applied Physics 2012, 112, 134404-523.

17. Shivola, A. H. IEEE Transactions on Geoscience \& Remote Sensing 1989, 27, 403 - 415. 